\title{
Wavefront reconstruction by adding modulation capabilities of two liquid crystal devices
}

\author{
Raúl Tudela \\ Estela Martín-Badosa \\ Ignasi Labastida \\ Santiago Vallmitjana, MEMBER SPIE \\ Artur Carnicer \\ Universitat de Barcelona \\ Departament de Física Aplicada i Optica \\ Avda. Diagonal 647 \\ E08028 Barcelona, Spain \\ E-mail: raul@fao.ub.es
}

\begin{abstract}
We analyze the behavior of complex information in the Fresnel domain, taking into account the limited capability to display complex values of liquid crystal devices when they are used as holographic displays. To do this analysis we study the reconstruction of Fresnel holograms at several distances using the different parts of the complex distribution. We also use the information adjusted with a method that combines two configurations of the devices in an adding architecture. The results of the error analysis show different behavior for the reconstructions when using the different methods. Simulated and experimental results are presented. (๑) 2004 Society of Photo-Optical Instrumentation Engineers. [DOI: 10.1117/1.1803551]
\end{abstract}

Subject terms: Fresnel diffraction; wavefront propagation; holography; liquid crystal devices; optical image processing.

Paper 0431102 received Nov. 25, 2003; revised manuscript received Apr. 21, 2004; accepted for publication May 5, 2004.

\section{Introduction}

In many optical applications, it is necessary to use complex information, which must be accurately controlled and displayed in real time. The use of liquid crystal devices (LCDs) as spatial light modulators (SLMs) has become a generalized way to obtain real-time manipulation of wavefronts, which implies the use of complex functions to modify the amplitude and the phase of a wave. Most of the present LCDs provide a wide range of operating modes, but none of them enables us to completely display the full complex plane. This limitation makes it necessary to develop some strategies to achieve full complex modulation. A first approach could be to use only one display where the complex information could be codified, using classical holographic methods ${ }^{1}$ or new approaches. ${ }^{2-4}$ These methods entail a loss of resolution when displaying the information on the devices. A second strategy is to couple two SLMs to obtain full complex modulation, taking into account the coupling optical setup and the modulation capabilities of each device. Juday and Florence ${ }^{5}$ and Gregory et al. ${ }^{6}$ proposed the two common architectures using two LCDs, one combining amplitude and phase as a product, which can be achieved with different architectures and devices, ${ }^{7-9}$ and another one in which the real and imaginary parts are combined as an addition. ${ }^{10}$

In this paper, we use the additive architecture to reconstruct Fresnel holograms by coupling two devices with arbitrary configurations, other than real-only or imaginaryonly ones. First, we present a method to represent a complex number as the sum of two phases. Then we propose an alternative approach by adding two complex numbers that are restricted to the values of LCD operating curves. We present simulated results of this method to adjust complex functions to the combination of different configurations in an adding architecture. We also study Fresnel hologram reconstructions when using only part of the com- plex information, or using the full complex distribution adjusted to actual configurations. Finally, we present some experimental results using two video graphics array (VGA) displays working with different configurations and the conclusions.

\section{Addition of Two LCDs to Represent Complex Information}

Complex numbers are usually represented as the addition of the real and imaginary parts or as the product of the amplitude and the phase:

$z=a+i b=M e^{i \theta}$.

Another possible way to write a complex number is as the addition of two phases:

$z=e^{i \phi_{1}}+e^{i \phi_{2}}$

This decomposition is valid when the amplitude of the complex number is smaller than two $(M \leqslant 2)$, as two complex numbers with amplitude equal to one are added. The relationship between the phases $\phi_{1}$ and $\phi_{2}$ in Eq. (2) and the real and imaginary parts in Eq. (1) is

$$
\begin{aligned}
& \phi_{1}=\arctan \frac{b}{a}+\arccos \left(\frac{a^{2}+b^{2}}{4}\right)^{1 / 2} \\
& \phi_{2}=\arctan \frac{b}{a}-\arccos \left(\frac{a^{2}+b^{2}}{4}\right)^{1 / 2} .
\end{aligned}
$$

The latter approach enables the representation of complex information by adding phase-only information displayed on two LCDs used as SLMs. Depending on the characteristics of the devices, the addition of two wavefronts is obtained using a different architecture. Figure 1 

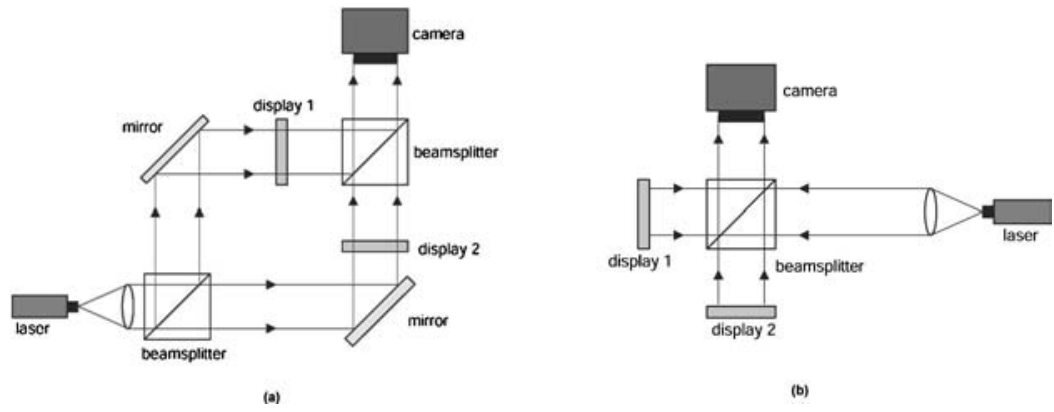

Fig. 1 Architectures to obtain the addition of two wavefronts displayed on LCDs (a) for transmissive devices and (b) for reflective devices.

shows the architectures for the addition of two wavefronts for transmissive and reflective SLMs. In both cases, the result in the observing plane is the addition of the propagation of the distributions displayed on each LCD. To obtain the addition of what is directly displayed on the devices, without considering the propagation, it would be necessary to use a lens system for imaging the LCDs onto the camera plane. The problem appears when the two phases that represent a complex number must be displayed on real LCDs, which have limited capabilities and thus never give perfect (ideal) phase-only modulation (continuous phase values from 0 to $2 \pi$ with amplitude 1$)$. Therefore, the computed phase values will not correspond exactly to the limited complex values that can be modulated by the actual devices, and thus, the displayed will differ from the computed information.

A different approach that takes into account the modulation capabilities of the devices is to directly adjust each computed complex value to a sum of two complex numbers that belong to the modulation curves (MC) of the devices.

$z \simeq z_{1}+z_{2} \quad$ with $z_{1} \in \mathrm{MC}_{1} \quad$ and $z_{2} \in \mathrm{MC}_{2}$.

Then each modulator can work in a suitable configuration and by adding the modulation curves of the two devices we obtain a new curve that covers more area of the complex plane than using the two separated curves.

We applied these techniques using three configurations of EPSON VGA video projector EMP-3000 panels, which can modulate 256 different gray levels: the first one [Fig. $2(\mathrm{a})]$ is a phase-mostly (PM) configuration with a maximum of phase modulation of $1.60 \pi$ and a contrast ratio of $5.4: 1$; the second configuration is a $0-\pi$ phase-only (PO) curve with a maximum of phase modulation of $0.98 \pi$ and a contrast ratio of 1.1:1 [Fig. 2(b)]; the last curve is a high contrast (HC) configuration with a maximum of phase modulation of $0.75 \pi$ and a contrast ratio of 124:1 [Fig. 2(c)].

In the first technique ("separated") we separate the complex information to be displayed into the two phases obtained using Eq. (2), then we use these configurations to adjust each of the computed phases to one of the curves, and finally, we add the two parts. This gives us nine different combinations using the three curves shown in Fig. 2.

In the second technique ("combined") we combine two of the three curves of Fig. 2 by adding each point of one configuration with all the points of another one. By doing this, we obtain the six added curves shown in Fig. 3. In these curves the $256^{2}$ values that can be modulated by using two devices in an adding configuration are represented. Then we adjust the full complex information directly to one of these "dense" distributions.

The method used to adjust the complex information to the curves is as follows. First, the complex values are normalized to the maximum amplitude of the curve, and then each complex number is adjusted to its nearest point in the curve by using a minimum Euclidean distance (MED) criterion. The combination that covers the normalized complex plane more densely will give better results when representing a complex function.

To study the capability of the different combinations to modulate the complex plane for the two techniques, we used a test distribution that represents the unity complex plane. Figure 4 shows the images (256 gray levels) of the amplitude from $M=0$ (black in the center) to $M=\sqrt{2}$

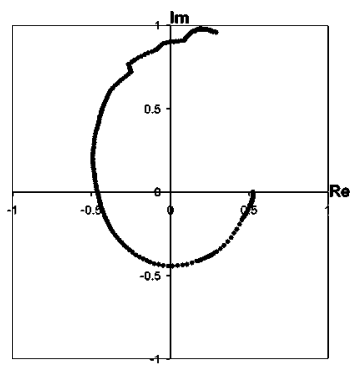

(a)

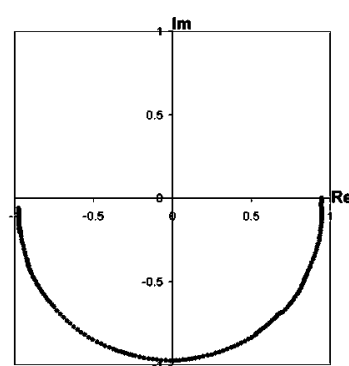

(b)

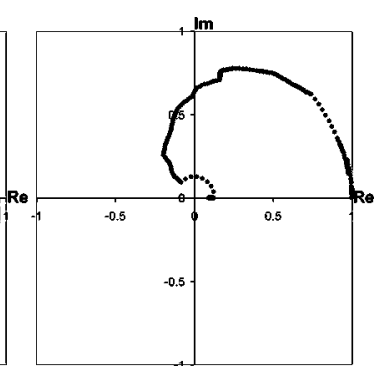

(c)

Fig. 2 Modulation curves of the VGA devices: (a) PM, (b) $0-\pi$ PO, and (c) HC configurations. 


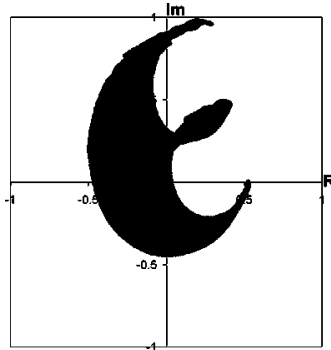

(a)

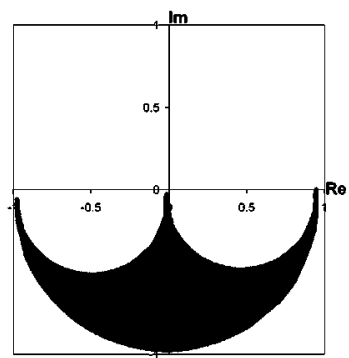

(d)

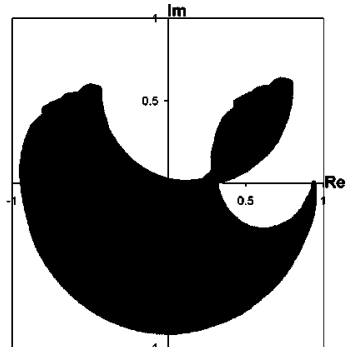

(b)

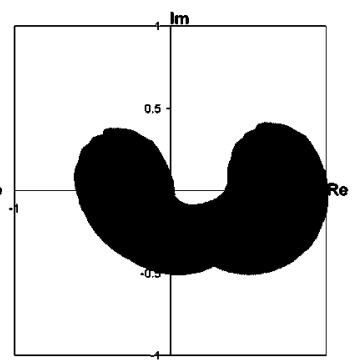

(e)

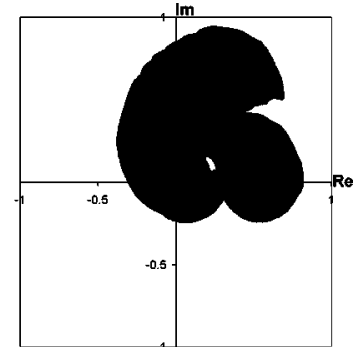

(c)

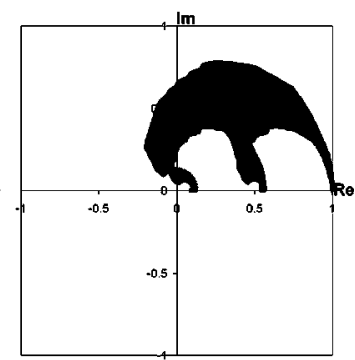

(f)

Fig. 3 Accessible values with the addition of two configuration curves: (a) PM+PM, (b) PM+PO, (c) $\mathrm{PM}+\mathrm{HC}$, (d) $\mathrm{PO}+\mathrm{PO}$, (e) $\mathrm{PO}+\mathrm{HC}$, and (f) $\mathrm{HC}+\mathrm{HC}$.

(white in the corners) [Fig. 4(a)], the phase from $\theta=0$ (black line) to $\theta=2 \pi$ (white line) [Fig. 4(b)], the real part from $a=-1$ (black column) to $a=1$ (white column) [Fig. $4(\mathrm{c})]$, and the imaginary part from $b=-1$ (black row) to $b=1$ (white row) [Fig. 4(c)]. For the separated technique, we calculated the two phases that, following Eq. (2), give the complex number at each point of the test distribution. Then, each phase is adjusted to one of the three curves of Fig. 2. We computed the average distance between the ideal distribution and the results obtained when the two phases adjusted to the curves are added, using the nine possible combinations. These results are shown in the left part of Fig. 5, where the names of the configurations used for phases $\phi_{1}$ and $\phi_{2}$, respectively, are indicated. The results obtained for the second technique, in which the ideal dis- tribution is directly adjusted to one of the six curves obtained combining two of the configurations, are shown on the right. The adjustment of the unity complex plane to each of the possible combination can be also separated into its real, imaginary, amplitude, and phase parts [see Figs. 4(e) to 4(h), where we show the images of the unity complex plane adjusted to the $\mathrm{PM}+\mathrm{HC}$ combination]. The quality of the adjustment is then quantified with the root mean squared (rms) error [see Eq. (5)] between the image of each part of the adjusted distribution and the corresponding ideal part. These results are shown in the graph of Fig. 6 , where we can see the differences between the different combinations, for the real, imaginary, amplitude, and phase parts, in the case of separating the distribution into phases, on the left (first technique), and when adjusting the com-

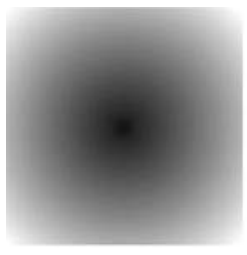

(a)

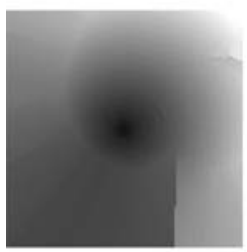

(e)

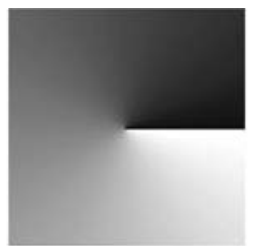

(b)

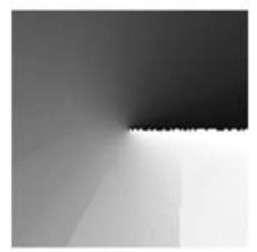

(f)

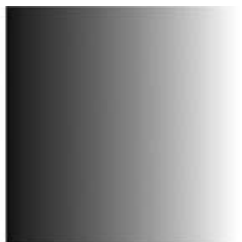

(c)

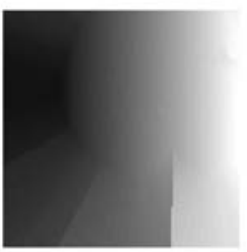

(g)

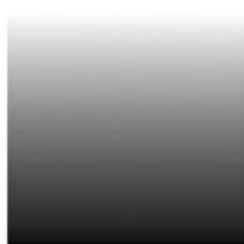

(d)

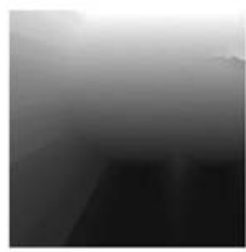

(h)

Fig. 4 Images of the ideal unity complex plane distribution: (a) amplitude, (b) phase, (c) real part, and (d) imaginary part, and images of the unity complex plane distribution adjusted to the $\mathrm{PM}+\mathrm{HC}$ combination: (e) amplitude, (f) phase, (g) real part, and (h) imaginary part. 


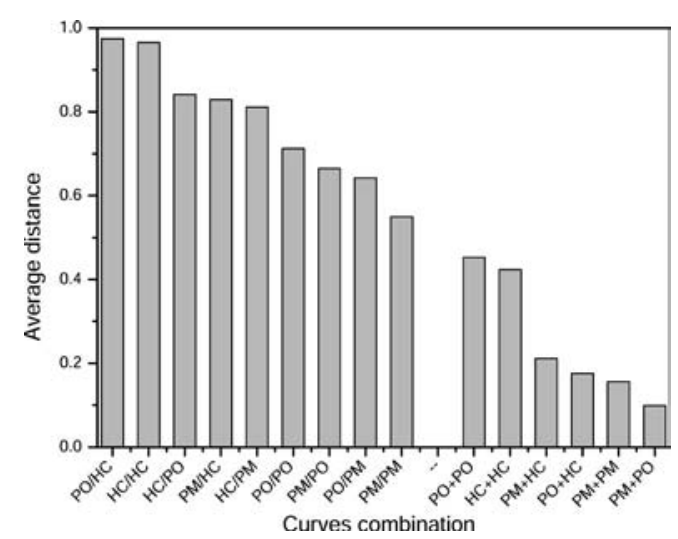

Fig. 5 Average distance between the ideal unity complex plane distribution and the obtained after adjustment: on the left, two phases are separately adjusted to two configuration curves; on the right, complex values are adjusted to combined curves.

plex plane to a combined curve, on the right (second technique).

$$
\begin{aligned}
\mathrm{rms}= & \left\{\frac{1}{N} \sum_{i=1}^{N}\left[A_{\text {adjusted }}(i)-A_{\text {ideal }}(i)\right]^{2}\right\}^{1 / 2} \text { with } \\
& N=\text { number of pixels. }
\end{aligned}
$$

The results obtained when adjusting the two phases separately are worse than the results of the method that combines two curves, as the values that can be modulated by the devices differ from the phases computed theoretically. From the results on the left side of Fig. 5, we can see that the worst reconstructions are obtained when using $\mathrm{HC}$ and PO curves, as they have less phase modulation. The best results with this technique are obtained using two PM curves, as they provide the greatest phase modulation with a low amplitude contrast ratio. Using the PM curve together with the $\mathrm{HC}$ configuration gives worse reconstructions than using the PM curve with the PO configuration, as the $\mathrm{HC}$ curve introduces more amplitude modulation than the PO curve, while providing similar phase modulation.

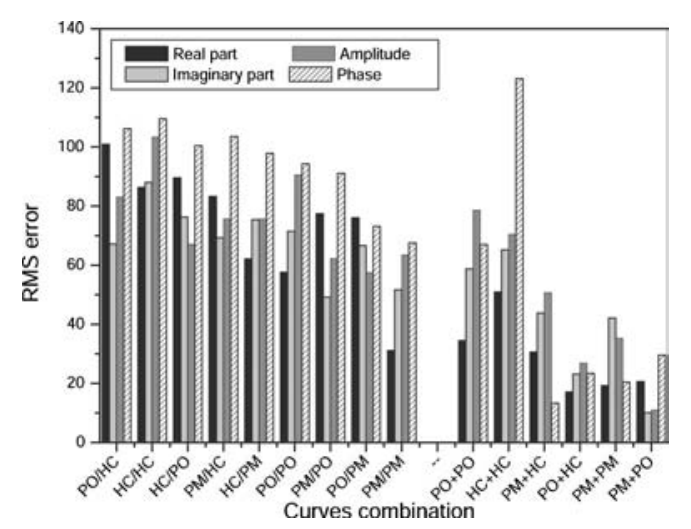

Fig. 6 The rms errors between the ideal unity complex plane images (real and imaginary parts, amplitude and phase) and the corresponding distributions obtained after adjustment: on the left, two phases are separately adjusted to two configuration curves; on the right, complex values are adjusted to combined curves.
The results obtained when using the combined technique are always better than for the first separated technique. The lowest errors are obtained for the PM+PM [Fig. 3(a)] and $\mathrm{PM}+\mathrm{PO}[\mathrm{Fig} .3(\mathrm{~b})]$ combinations, which have values that are distributed more uniformly over the complex plane, while the highest errors are obtained using the $\mathrm{PO}+\mathrm{PO}$ [Fig. 3(d)] and HC+HC [Fig. 3(f)] combinations, in which the accessible points are concentrated in a reduced zone of the plane. In this paper, we have chosen the PM+HC [Fig. 3 (c) ] combination, which can de used experimentally using two displays from the Epson videoprojector. This configuration has an intermediate behavior, and it is achieved by adding PM and HC operating configurations of the LCDs.

\section{Reconstruction of Fresnel Holograms}

We used the PM+HC combination to reconstruct Fresnel holograms as examples of a complex function to be displayed using an adding architecture. The holograms are computed by backpropagating an object $f(x, y)$ to a distance $-d$, using

$$
\begin{aligned}
U(x, y,-d)= & \text { Fresnel }[f(x, y),-d] \\
= & \frac{\exp (-i 2 \pi d / \lambda)}{i \lambda d} \int_{-\infty}^{\infty} f\left(x^{\prime}, y^{\prime}\right) \\
& \times \exp \left\{-\frac{i \pi}{\lambda d}\left[\left(x-x^{\prime}\right)^{2}\right.\right. \\
& \left.\left.+\left(y-y^{\prime}\right)^{2}\right]\right\} \mathrm{d} x^{\prime} \mathrm{d} y^{\prime} .
\end{aligned}
$$

This complex distribution $U(x, y,-d)$ is then adjusted to the PM+HC combined map. The object to be reconstructed is the University of Barcelona logo shown in Fig. 7 (a), which represents the shadow of a building and the letters $U$ and B. The Fresnel hologram to reconstruct this image at $849 \mathrm{~mm}$ is calculated with Eq. (6). Figure 7(b) corresponds to the simulated reconstruction obtained when adjusting the hologram using the accessible complex values of the PM+HC map and shows a good agreement with the original object. We also studied the quality of the reconstructions when using the separated technique, in which every complex value is written as the addition of two phases, $\phi_{1}$ and $\phi_{2}$, which are adjusted separately to different configuration curves (PM and $\mathrm{HC}$ in this case). Figures 7(c) ( $\phi_{1}$ adjusted to PM and $\phi_{2}$ adjusted to $\mathrm{HC}$ ) and 7(d) ( $\phi_{1}$ adjusted to $\mathrm{HC}$ and $\phi_{2}$ adjusted to PM) show much noisier reconstructions than those obtained with the combined method [Fig. 7(b)]. If a single configuration is used to adjust the complex values, we obtain the reconstructions shown in Figs. 7(e) for the PM configuration and 7(f) for the HC curve. Again, the quality is very poor compared with the results obtained using the combined $\mathrm{PM}+\mathrm{HC}$ configuration. This demonstrates that it is better to combine two modulators together than to use a single one, when representing complex information.

We also studied the quality of the reconstructions at different distances by computing the rms error. To do this, the original object to be reconstructed [Fig. 7(a)] was backpropagated to a distance $-d$; then, the obtained complex 


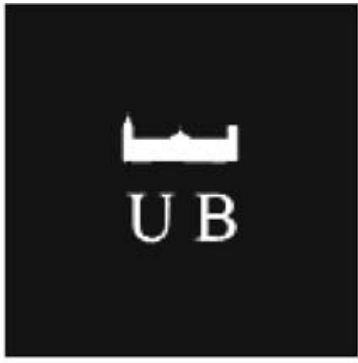

(a)

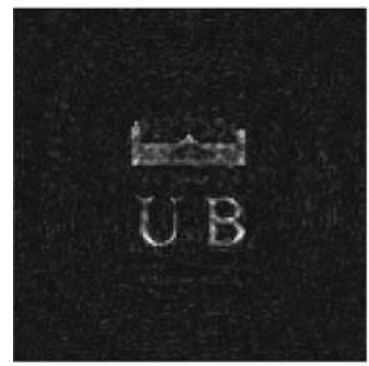

(c)

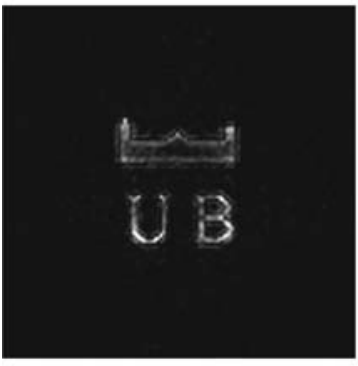

(e)

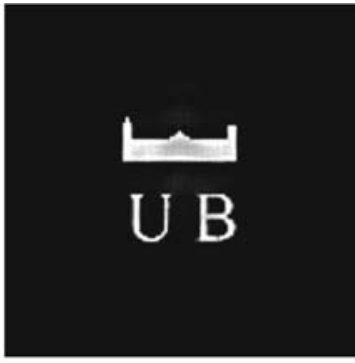

(b)

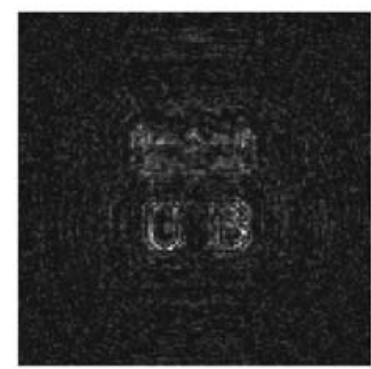

(d)

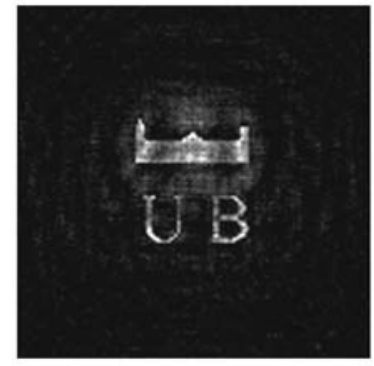

(f)
Fig. 7 Images of (a) the original object to be reconstructed (logo with building and letters) and the simulated reconstructions at 849 $\mathrm{mm}$ using (b) the combined method with the PM+HC combination, (c) the phase addition with phase $\phi_{1}$ adjusted to the PM curve and $\phi_{2}$ to the $\mathrm{HC}$ curve, (d) the phase addition with phase $\phi_{1}$ adjusted to the $\mathrm{HC}$ curve and $\phi_{2}$ to the PM curve, (e) only the PM curve, and (f) only the $\mathrm{HC}$ curve. distribution was separated in its different parts: real, imaginary, amplitude, and phase. In the next step, each part and the full complex distribution can be adjusted to a configuration curve or can be used without adjustment, and finally, each of them was propagated to a distance $d$, where the object was reconstructed. The rms error was computed by comparing the reconstructed amplitudes with the original one. Figure 8(a) shows the results for 2000 calculated propagation distances between 0 and $3000 \mathrm{~mm}$, using only part of the ideal complex information (real or imaginary, amplitude, or phase), without adjusting to any curve. The error in the reconstructions using only the phase of the wavefront (which contains high-frequency information) is the highest one, and it is practically constant. This is because the phase contains high-frequency information so that the bulk of the object is lost. The error in the amplitude reconstruction (which contains low-frequency information) increases with the distance, and it is small for short distances as the behavior predicted by the geometrical optics prevails. The errors of the reconstructions using only the real or the imaginary parts oscillate between the two solid black lines shown in the graph of Fig. 8(a). In Fig. 8(b) there is a detail of these oscillations in a small zone around $849 \mathrm{~mm}$; they have a period of $\lambda / 2$ caused by the constructive or destructive interferences for the real or imaginary parts, depending on the distance, as explained in detail in Ref. 10. The difference between the lower and top errors of the oscillations depends on the image used to do the reconstructions.

In Fig. 9(a) we plot the rms errors of the reconstructed amplitudes using the combined method with the $\mathrm{PM}+\mathrm{HC}$ configuration for 2000 propagation distances between 0 and $3000 \mathrm{~mm}$. The error oscillates between the two solid lines and, even in the worst case, always remains smaller than when using only one part of the ideal complex function. The oscillations are shown in detail in Fig. 9(b), where the amplitude error is plotted in a small zone around 849 $\mathrm{mm}$. These oscillations are due to the phase factor $\exp (-i 2 \pi d / \lambda)$ of the Fresnel transform [Eq. (6)], which introduces a global phase to all the points of the complex distribution. This phase depends on the distance, with a period of $\lambda$, and its effect is to rotate the points of the distribution of Eq. (6) on the complex plane. When these

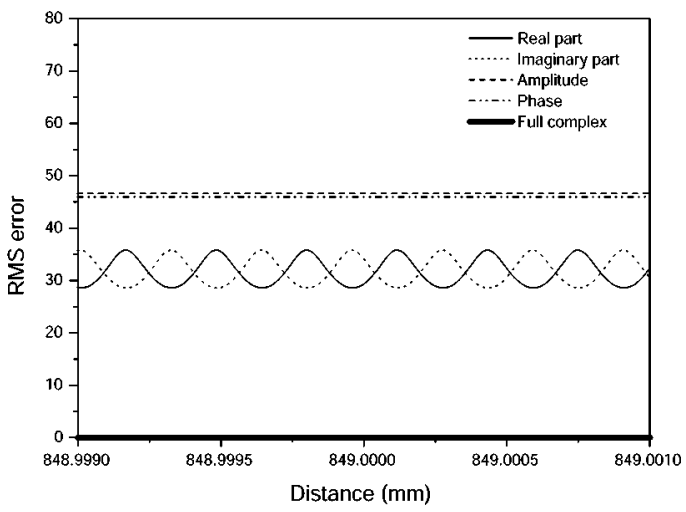

(b)

Fig. 8 (a) The rms errors for the reconstructed amplitude of the logo image [see Fig. 7(a)] at distances between 0 and $3000 \mathrm{~mm}$, using the different ideal parts, and (b) detail of the oscillations of the rms errors around $849 \mathrm{~mm}$. 


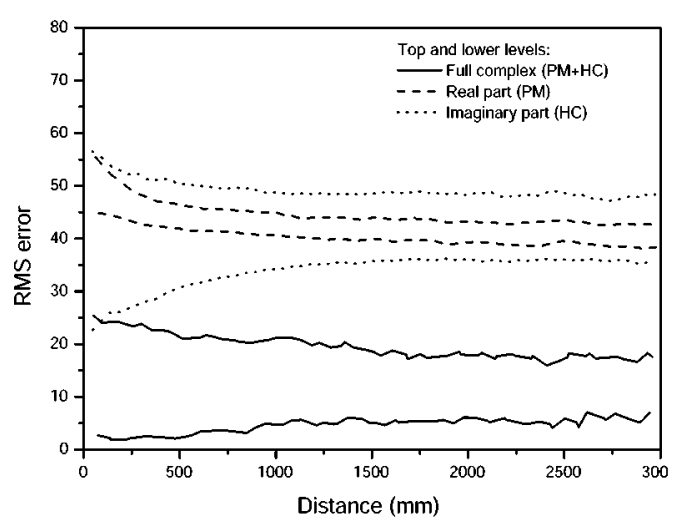

(a)

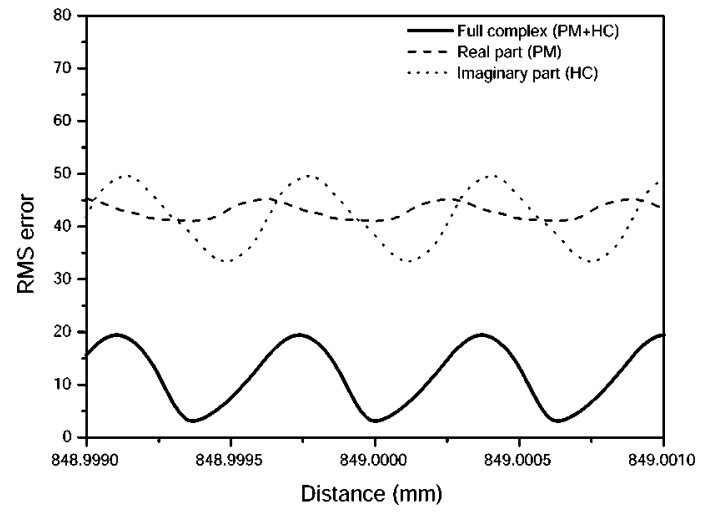

(b)

Fig. 9 (a) The rms errors for the reconstructed amplitude of the logo image [see Fig. 7(a)] at distances between 0 to $3000 \mathrm{~mm}$, using the combined method with the PM+HC combination, or just the real or imaginary part separately adjusted to the PM and $\mathrm{HC}$ curves, respectively, and (b) detail of the oscillations of the rms errors around $849 \mathrm{~mm}$.

complex values are located in a zone that is not covered by the configuration curve, the error of the reconstruction increases; the error is lower when the distribution falls in a region well covered by the curve. In all cases, the lowest error with the combined method is below the lowest error using part of the complex information in the ideal case. In Fig. 9, we also plotted the errors of the amplitude object reconstructions using only the real part adjusted to the PM configuration and using only the imaginary part adjusted to the $\mathrm{HC}$ curve. In these cases, we can see that the error when adjusting the data to a single operating curve is larger than in the ideal case. We can also see how the oscillations of these adjustments are related to the oscillations of the combined case.

\section{Experimental Results}

We designed an optical setup using two VGA displays removed from an Epson EMP-3000 videoprojector. It consists of the two LCDs, with $512 \times 512$ pixels and a pixel pitch of $42 \mu \mathrm{m}$, arranged in an interferometric configuration as shown in Fig. 1(a). We used a He-Ne laser source of $632.8 \mathrm{~nm}$ and a CCD camera that can be placed in the position where the object is reconstructed, at the backpropagated distance of the computed Fresnel hologram. We reconstructed the object shown in Fig. 7(a) at the same distance that was performed in the simulations. Figure 10 shows the experimental reconstruction using the full complex information adjusted to the $\mathrm{PM}+\mathrm{HC}$ configuration, using the combined method.

We also used the combined method with the PM+HC curve to adjust a hologram computed to reconstruct different objects at different distances. We used the letters in Fig. 7(a) as the object to be displayed in two positions and reconstructed at two different distances. In Fig. 11(a) we can see the reconstruction at $849 \mathrm{~mm}$, where the lower letters are recovered while the top letters are defocused, and the inverse situation in Fig. 11(b), where the reconstruction is carried out at $867 \mathrm{~mm}$. This illustrates the possibility of reconstructing volume information from a single complex distribution, where it is important to have a low error for all the distances. This can be achieved using two coupled devices.

The loss of quality of the reconstructed images is due to experimental difficulties such as nonuniform illumination, noise introduced by the polarizing elements used to obtain the different configurations of the modulators, the possible lack of parallelism between the panels, and misalignments in the optical setup. To see the importance of having the two devices perfectly aligned we calculated the rms error between the original image of the logo and the reconstructions when one device is displaced with respect to the other a certain number of pixels in the horizontal and vertical directions. In the graph of Fig. 12 we can see that by displacing one of the panels only 1 pixel, the error of the reconstruction increases considerably, and then it increases uniformly with the number of displaced pixels.

\section{Conclusion}

We analyzed the behavior of complex information in the Fresnel domain taking into account the limited capability of LCDs to display complex transmittance values. We proposed a method to represent complex functions as the sum of two phases. This approach is not suitable to be used directly with LCDs that are unable to modulate phase ideally. To adapt this method to actual configurations we de-

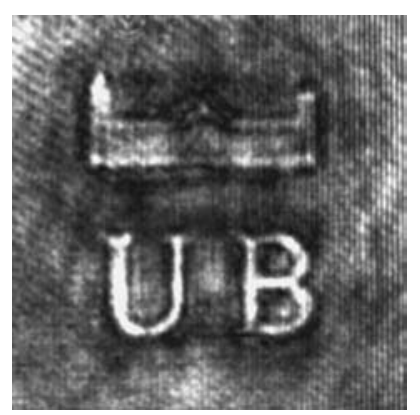

Fig. 10 Experimental reconstruction of the image of the logo [Fig. $7(\mathrm{a})]$ at $849 \mathrm{~mm}$ using the combined method with the PM+HC curve. 


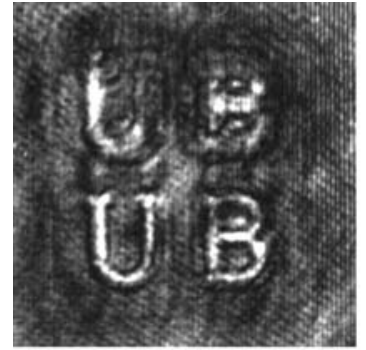

(a)

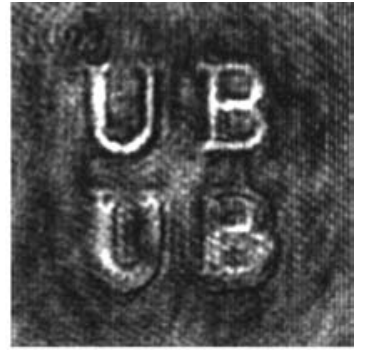

(b)
Fig. 11 Experimental reconstruction of two pairs of UB letters: (a) at $849 \mathrm{~mm}$, which focus the lower letters, and (b) at $867 \mathrm{~mm}$, which focus the top letters, using the combined method with the PM+HC curve.

vised an alternative to adjust a complex function to a sum of two complex numbers, which are restricted to the operating curves of two modulators placed in an adding architecture. To analyze this procedure we considered three actual configurations of VGA panels. We studied the quality of the adjustment of the ideal unity complex plane for the nine different ways to adjust the two phases using the three curves and the six possible combined curves obtained adding the three configurations. For this purpose, we computed the Euclidean distance between points and the rms error between the images of the different parts (amplitude, phase, real part, and imaginary part) of the ideal and the adjusted unity complex planes. The best results were obtained using the method that adds two configurations, for the combined curve that covers more densely and uniformly the normalized complex plane.

We computed the reconstruction of Fresnel holograms at several propagation distances using the different parts of the complex distribution (real and imaginary parts, amplitude, and phase) as well as using the full complex information adjusted to the combined configuration using the PM and the HC curves. The rms error between the amplitudes of these reconstructions and the original amplitude was calculated to evaluate the quality of the information displayed. The error analysis results showed different behavior for the

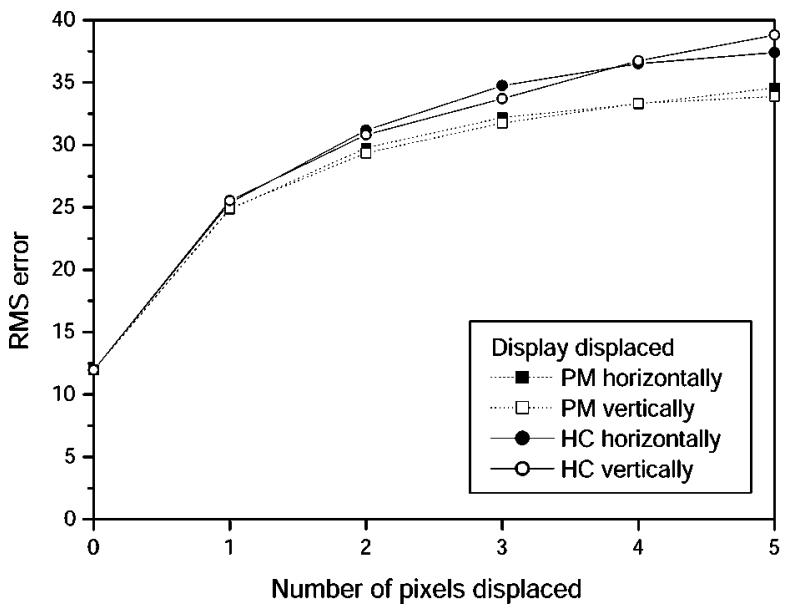

Fig. 12 Graph of the rms errors for the reconstructed amplitude of the logo image at $849 \mathrm{~mm}$ when one of the devices is displaced respect the other. reconstructions using the different parts of the ideal complex distribution. When using only the phase, we obtain a constant but poor reconstruction for all the propagation distances, while with the amplitude, the quality of the reconstructions is higher for short distances and decreases with the distance, giving worse reconstructions than with the phase. On the other hand, the real and the imaginary parts have a complementary behavior: their reconstruction quality oscillates, with a period of half the wavelength, between a top and a lower level. When the real part gives a maximum of the rms error, the imaginary part gives a minimum and vice versa. In the case of using the actual configurations of the modulators with the combined method, the rms error also oscillates and gives better reconstructions than using only part of the ideal complex function. The experimental results using this method in an optical setup present good agreement with the simulated results, and show that it is possible to obtain full complex modulation by combining two configurations of the modulators, such that greater part of the complex plane is densely covered when added together.

\section{Acknowledgments}

This paper was partially funded by the CICYT (Comisión Interministerial de Ciencia y Tecnología) through Project No. DPI 2001-3365, and by the CIRIT (Comissió Interdepartamental de Recerca i Tecnologia) through Project No. 2001SGR00079.

\section{References}

1. G. Tricoles, "Computer generated holograms: an historical review," Appl. Opt. 26, 4351-4360 (1987).

2. S. Serati and K. Bauchert, "Sampling technique for achieving full unit-circle coverage using a real-axis spatial light modulator," in $O p$ tical Pattern Recognition X, D. P. Casasent, Ed., Proc. SPIE 3715, 112-119 (1999).

3. P. Birch, R. Young, C. Chatwin, M. Farsari, D. Budgett, and J. Richardson, "Fully complex optical modulation with an analogue ferroelectric liquid crystal spatial light modulator," Opt. Commun. 175, 347-352 (2000).

4. J. A. Davis, K. O. Valadéz, and D. M. Cottrell, "Encoding amplitude and phase information onto a binary phase-only spatial light modulator," Appl. Opt. 42, 2003-2008 (2003).

5. R. D. Juday and J. M. Florence, "Full complex modulation with two one-parameter slm's," in Wave Propagation and Scattering in Varied Media II, V. K. Varadan, Ed., Proc. SPIE 1558, 499-504 (1991).

6. D. A. Gregory, J. C. Kirsch, and E. C. Tam, "Full complex modulation using liquid-crystal televisions," Appl. Opt. 31, 163-164 (1992).

7. J. Amako, H. Miura, and T. Sonehara, "Wave-front control using liquid-crystal devices," Appl. Opt. 32, 4323-4329 (1993).

8. L. G. Neto, D. Roberge, and Y. Sheng, "Full-range, continuous, complex-modulation by the use of two coupled-mode liquid-crystal televisions," Appl. Opt. 35, 4567-4576 (1996).

9. R. Tudela, I. Labastida, E. Martín-Badosa, S. Vallmitjana, I. Juvells, and A. Carnicer, "A simple method for displaying Fresnel holograms on liquid crystal panels," Opt. Commun. 214, 107-114 (2002).

10. R. Tudela, E. Martín-Badosa, I. Labastida, S. Vallmitjana, I. Juvells, and A. Carnicer, "Full complex Fresnel holograms displayed on liquid crystal devices," J. Opt. A, Pure Appl. Opt. 5, S189-S194 (2003).

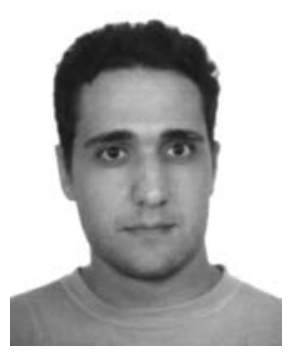

Raúl Tudela studied physics at the Universitat de Barcelona, where he received his BS and MSc degrees in 1998 and 2000. $\mathrm{He}$ is an assistant professor with the Grup d'Optica Física i Fotònica at the Universitat de Barcelona, and he is working on his doctoral thesis. His research interests include computer-generated holograms, spatial light modulators, and image processing. $\mathrm{He}$ is a member of the Societat Catalana de Física (Catalan Physics Society). 


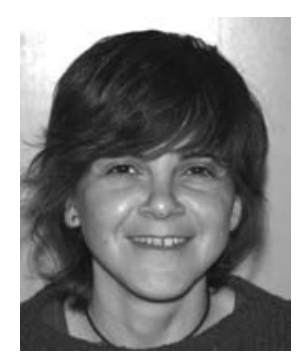

Estela Martín-Badosa received her BSc, MSc, and PhD degrees in physics in 1992, 1994, and 1998, respectively, and her BSc degree in electronic engineering in 2003, all from the University of Barcelona. She is currently an assistant professor with the Grup d'Optica Física i Fotònica (Departament de Física Aplicada i Optica, Universitat de Barcelona). Her research interests include spatial light modulation, optical pattern recognition, 3-D image processing, and holography. She is a member of the Societat Catalana de Física (Catalan Physics Society).

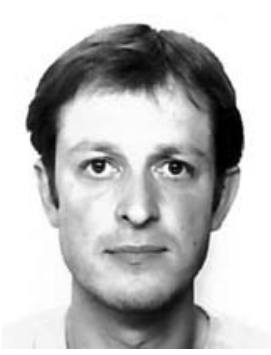

Ignasi Labastida received his BS, MS, and $\mathrm{PhD}$ degrees in physics from Universitat of Barcelona, in 1994, 1997, and 2000 , respectively. He is currently collaborating with the Grup d'Optica Física i Fotònica at the Universitat de Barcelona. His research interests include optical pattern recognition, spatial light modulators, and computer-generated holograms. He is a member of the Societat Catalana de Física (Catalan Physics Society).

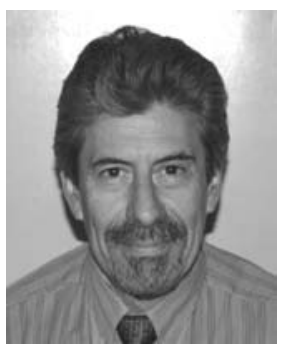

Santiago Vallmitjana received his MS degree in physics in 1976 and his PhD degree in 1980 from the University of Barcelona. He joined Optics Laboratory in 1974 and has been working on measurement of the optical transfer function, image quality analysis, holography, optical information processing, and applications on biological images. He is teaching optics and conducting postgraduate research at the University of Baroelona. Vallmitjana is the author or coauthor of more than a hundred scientific papers, and his main current interests are real-time 3-D optical pattern recognition, optical computing, and beam shaping by spatial light modulators. He is a member of SCF (Societat Catalana de Física), SCHCT (Societat Catalana d'Història de la Ciència i de la Tècnica), SEDO (Sociedad Española de Optica), AERFAl (Asociación Española de Reconocimiento de Formas y Análisis de Imágenes), EOS, and SPIE.

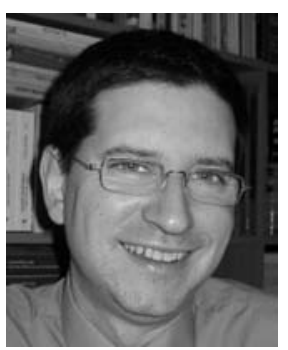

Artur Carnicer received his $\mathrm{PhD}$ degree in physics in 1993 from the Universitat de Barcelona, where he is currently an associate professor. His research focuses on digital holography, optical image processing, and holographic optical tweezers and he has published more than 40 papers in these fields. 